\title{
CONVEX REAL PROJECTIVE STRUCTURES ON CLOSED SURFACES ARE CLOSED
}

\author{
SUHYOUNG CHOI AND WILLIAM M. GOLDMAN
}

(Communicated by Jonathan M. Rosenberg)

\begin{abstract}
The deformation space $\mathfrak{C}(\Sigma)$ of convex $\mathbb{R P}^{2}$ - structures on a closed surface $\Sigma$ with $\chi(\Sigma)<0$ is closed in the space $\operatorname{Hom}(\pi, \operatorname{SL}(3, \mathbb{R})) / \operatorname{SL}(3, \mathbb{R})$ of equivalence classes of representations $\pi_{1}(\Sigma) \rightarrow \operatorname{SL}(3, \mathbb{R})$. Using this fact, we prove Hitchin's conjecture that the contractible "Teichmüller component" (Lie groups and Teichmüller space, preprint) of $\operatorname{Hom}(\pi, \operatorname{SL}(3, \mathbb{R})) / \mathrm{SL}(3, \mathbb{R})$ precisely equals $\mathfrak{C}(\Sigma)$.
\end{abstract}

Let $\Sigma$ be a closed orientable surface of genus $g>1$ and $\pi=\pi_{1}(\Sigma)$ its fundamental group. A convex $\mathbb{R} \mathbf{P}^{2}$-structure on $M$ is a representation (uniformization) of $M$ as a quotient $\Omega / \Gamma$ where $\Omega \subset \mathbb{R} \mathbf{P}^{2}$ is a convex domain and $\Gamma \subset \operatorname{SL}(3, \mathbb{R})$ is a discrete group of collineations of $\mathbb{R} P^{2}$ acting properly and freely on $\Omega$. (See [5] for basic theory of such structures.) The space of projective equivalence classes of convex $\mathbb{R P}^{2}$-structures embeds as an open subset in the space of equivalence classes of representations $\pi \rightarrow \operatorname{SL}(3, \mathbb{R})$. The purpose of this note is to show that this subset is also closed.

In [7], Hitchin shows that the space of equivalence classes of representations $\pi \rightarrow \mathrm{SL}(3, \mathbb{R})$ falls into three connected components: one component $C_{-1}$ consisting of classes of representations for which the associated flat $\mathbb{R}^{3}$-bundle over $\Sigma$ has nonzero second Stiefel-Whitney class; a component $C_{0}$ containing the class of the trivial representation; a component $C_{1}$ diffeomorphic to a cell of dimension $16(g-1)$, which he calls the "Teichmüller component." While $C_{-1}$ can be distinguished from $C_{0}$ and $C_{1}$ by a topological invariant [3, 4], no characteristic invariant distinguishes representations in the Teichmüller component from those in $C_{0}$. The Teichmüller component is defined as follows. Using the Klein-Beltrami model of hyperbolic geometry, a hyperbolic structure on $\Sigma$ is a special case of a convex $\mathbb{R} P^{2}$-structure $\Omega / \Gamma$ where $\Omega$ is the region bounded by a conic. In this case $\Gamma$ is conjugate to a cocompact lattice in $\mathrm{SO}(2,1) \subset \mathrm{SL}(3, \mathbb{R})$. The space $\mathfrak{T}(\Sigma)$ of hyperbolic structures ("Teichmüller

Received by the editors August 16, 1991 and, in revised form, October 9, 1991; presented at the first joint meeting of the American Mathematical Society and the London Mathematical Society in Cambridge, England, on July 1, 1992.

1991 Mathematics Subject Classification. Primary 57M50, 53A20; Secondary 53C15, 58D27.

The first author's research was partially supported by a grant from TGRC-KOSEF and the second author's research was partially supported by University of Maryland Institute of Advanced Computer Studies and National Science Foundation grant DMS-8902619. 
space") is a cell of dimension $6(g-1)$, which is a connected component of $\operatorname{Hom}(\pi, \mathrm{SO}(2,1)) / \mathrm{SO}(2,1)$. Regarding hyperbolic structures on $\Sigma$ as convex $\mathbb{R} \mathbf{P}^{2}$-structures embeds the Teichmüller space $\mathfrak{T}(\Sigma)$ inside $\mathfrak{C}(\Sigma)$. By [5], the space $\mathfrak{C}(\Sigma)$ of convex $\mathbb{R P}^{2}$-structures on a compact surface $\Sigma$ is shown to be diffeomorphic to a cell of dimension $16(g-1)$ and $\mathfrak{T}(\Sigma)$ embeds $C_{1}$ as the space of equivalence classes of embeddings of $\pi$ as discrete subgroups of $\mathrm{SO}(2,1) \subset \mathrm{SL}(3, \mathbb{R})$. Hitchin's component $C_{1}$ can thus be characterized as the component of $\operatorname{Hom}(\pi, \operatorname{SL}(3, \mathbb{R})) / \operatorname{SL}(3, \mathbb{R})$ containing equivalence classes of discrete embeddings $\pi \rightarrow \mathrm{SO}(2,1)$.

Theorem A. Hitchin's Teichmüller component $C_{1}$ equals the deformation space $\mathfrak{C}(\Sigma)$ of convex $\mathbb{R}^{2}$-structures on $\Sigma$.

In $[5,3.3]$ it is shown that the deformation space $\mathfrak{C}(\Sigma)$ is an open subset of $\operatorname{Hom}(\pi, \operatorname{SL}(3, \mathbb{R})) / \operatorname{SL}(3, \mathbb{R})$ containing $\mathfrak{T}(\Sigma)$ and hence an open subset of $C_{1}$. Let

$$
\Pi: \operatorname{Hom}(\pi, \operatorname{SL}(3, \mathbb{R})) \rightarrow \operatorname{Hom}(\pi, \operatorname{SL}(3, \mathbb{R})) / \operatorname{SL}(3, \mathbb{R})
$$

denote the quotient map. Also by $[5,3.2]$, every representation in $\Pi^{-1}(\mathfrak{C}(\Sigma))$ has image Zariski-dense in either a conjugate of $\operatorname{SO}(2,1)$ or $\operatorname{SL}(3, \mathbb{R})$ itself, and hence by $[5,1.12] \mathrm{SL}(3, \mathbb{R})$ acts properly and freely on $\Pi^{-1}(\mathfrak{C}(\Sigma))$. In particular, the restriction

$$
\Pi: \Pi^{-1}(\mathfrak{C}(\Sigma)) \rightarrow \mathfrak{C}(\Sigma)
$$

is a locally trivial principal $\operatorname{SL}(3, \mathbb{R})$-bundle. It follows that $\Pi^{-1}(\mathfrak{C}(\Sigma))$ is an open subset of $\operatorname{Hom}(\pi, \operatorname{SL}(3, \mathbb{R}))$. Thus Theorem $A$ is a corollary of

Theorem B. $\Pi^{-1}(\mathfrak{C}(\Sigma))$ is a closed subset of $\operatorname{Hom}(\pi, \operatorname{SL}(3, \mathbb{R}))$.

The rest of the paper is devoted to the proof of Theorem B. Arguments similar to the proof are given at the end of the first chapter of [1] and an analogous statement when $\Sigma$ is a pair-of-pants is proved in [5, $\S \S 4.4$ and 4.5] (where it is used in the proof of the main theorem). We feel there is a more comprehensive result for compact surfaces with boundary, with a geometric proof.

Assume that $\phi_{n}$ is a sequence of representations in $\operatorname{Hom}(\pi, \operatorname{SL}(3, \mathbb{R}))$ which converges to $\phi \in \operatorname{Hom}(\pi, \operatorname{SL}(3, \mathbb{R}))$ and that each $\Pi\left(\phi_{n}\right) \in \mathfrak{C}(\Sigma)$. Thus for each $n$, there exists a convex domain $\Omega_{n} \subset \mathbb{R} \mathrm{P}^{2}$ such that $\phi_{n}: \pi \rightarrow \operatorname{SL}(3, \mathbb{R})$ embeds $\pi$ onto a discrete group $\Gamma_{n}$ acting properly and freely on $\Omega_{n}$. Furthermore, as discussed in [5,3.2(1)], each $\Omega_{n}$ is strictly convex and has the property that the closure $\overline{\Omega_{n}}$ is a compact subset of an affine patch (the complement of a projective line) in $\mathbb{R} \mathbf{P}^{2}$.

We identify the universal covering of $\mathbb{R} \mathbf{P}^{2}$ with the 2-sphere $S^{2}$ of oriented directions in $\mathbb{R}^{3}$. Denote by $p: S^{2} \rightarrow \mathbb{R} \mathbf{P}^{2}$ the covering projection. The group

$$
\mathrm{SL}_{ \pm}(3, \mathbb{R})=\{A \in \mathrm{GL}(3, \mathbb{R}) \mid \operatorname{det}(A)= \pm 1\}
$$

acts on $S^{2}$ covering the action of $\operatorname{SL}(3, \mathbb{R})$ on $\mathbb{R} \mathbf{P}^{2}=S^{2} /\{ \pm 1\}$. A choice of positive definite inner product on $\mathbb{R}^{3}$ realizes $S^{2}$ as the unit sphere in $R^{3}$, and $d: S^{2} \times S^{2} \rightarrow \mathbb{R}$ denotes the distance function corresponding to the induced Riemannian metric. The geodesics in $S^{2}$ are arcs of great circles. If $\Omega \subset \mathbb{R} \mathbf{P}^{2}$ has the property that there exists an affine patch $A \subset \mathbb{R} \mathbf{P}^{2}$ such that $\Omega \subset$ $A$ is convex (with respect to the affine geometry on $A$ ), then we say that $\Omega$ 
is properly convex. In that case each component of $p^{-1}(\Omega)$ is convex in the corresponding elliptic geometry of $S^{2}$ and there exists a sharp convex cone in $\mathbb{R}^{3}$ whose projectivization equals $\Omega$. We shall also refer to a component of $p^{-1}(\Omega)$ as properly convex. (A sharp convex cone in an affine space $E$ is an open convex domain $\Omega \subset E$ invariant under positive homotheties and containing no complete affine line.)

Since an affine patch is contractible, $p^{-1}\left(\Omega_{n}\right)$ consists of two components each of which maps diffeomorphically to $\Omega_{n}$. Choose one of the components $\Omega_{n}^{\prime} \subset S^{2}$ for each $n$. Furthermore $\phi_{n}$ defines an effective proper action of the discrete group $\pi$ on $\Omega_{n}^{\prime}$ whose quotient is a convex $\mathbb{R} \mathbf{P}^{2}$-surface homeomorphic to $\Sigma$. Moreover, since $\pi$ is not virtually nilpotent and $\phi_{n}$ is a discrete embedding for each $n$, the limiting representation $\phi=\lim _{n \rightarrow \infty} \phi_{n}$ is also a discrete embedding (see, e.g., [6, Lemma 1.1]). In particular, the image $\Gamma$ of $\phi$ is torsionfree and not virtually abelian.

Since the space of compact subsets of $S^{2}$ is compact in the Hausdorff topology, we may (after extracting a subsequence) assume that the sequence $\overline{\Omega_{n}}$ ' converges (in the Hausdorff topology) to a compact subset $K \subset S^{2}$.

Lemma 1. $K$ is invariant under the image $\Gamma=\phi(\pi)$.

Proof. Suppose that $k \in K$ and $g \in \pi$. We show that $\phi(g) k \in K$. Let $\varepsilon>0$. Now $\phi_{n}(g)$ converges uniformly to $\phi(g)$ on $S^{2}$; thus there exists $N_{1}=N_{1}(\varepsilon)$ such that

$$
d\left(\phi_{n}(g) x, \phi(g) x\right)<\varepsilon / 2
$$

for $n>N_{1}$. Indeed the family $\phi_{n}(g)$ is uniformly Lipschitz for sufficiently large $n$-let $C$ be a Lipschitz constant, i.e.,

$$
d\left(\phi_{n}(g) x, \phi_{n}(g) y\right) \leq C d(x, y)
$$

for all $x, y \in S^{2}$ and $n$ sufficiently large, say $n>N_{2}$. Since $K$ is the Hausdorff limit of ${\overline{\Omega_{n}}}^{\prime}$, there exist $w_{n} \in{\overline{\Omega_{n}}}^{\prime}$ such that $w_{n} \rightarrow k$. Thus there exists $N_{3}=N_{3}(\varepsilon)$ such that $d\left(k, w_{n}\right)<\varepsilon /(2 C)$ for $n>N_{3}$. Putting these inequalities together, we obtain

$$
\begin{aligned}
d\left(\phi(g) k, \phi_{n}(g) w_{n}\right) & \leq d\left(\phi(g) k, \phi_{n}(g) k\right)+d\left(\phi_{n}(g) k, \phi_{n}(g) w_{n}\right) \\
& <\varepsilon / 2+C \varepsilon /(2 C)=\varepsilon
\end{aligned}
$$

for $n>\max \left(N_{1}, N_{2}, N_{3}\right)$. It follows that $\phi(g) k$ is the limit of $\phi_{n}(g) w_{n} \in$ ${\overline{\Omega_{n}}}^{\prime}$. Since the Hausdorff limit of ${\overline{\Omega_{n}}}^{\prime}$ equals $K$, it follows that $\phi(g) k \in K$, as claimed.

Furthermore each ${\overline{\Omega_{n}}}^{\prime}$ is convex in $S^{2}$. Since convex sets are closed in the Hausdorff topology, it follows that $K$ is also convex. (See [2] for more details.)

There are the following possibilities for $K$ (compare Choi [2]):

(1) $K$ is properly convex with nonempty interior.

(2) $K$ consists of a single point.

(3) $K$ consists of a line segment.

(4) $K$ is a great disk (i.e., a closed hemisphere).

We show that only case (1) can arise. The following lemma (whose proof we defer) is used to rule out the last three cases. 
Lemma 2. Suppose that $F$ is a nonabelian free group and $h: F \rightarrow \operatorname{SL}(2, \mathbb{R})$ is a homomorphism which embeds $F$ onto a discrete subgroup of $\operatorname{SL}(2, \mathbb{R})$. Then there exists $f \in F$ such that $h(f)$ has negative trace.

In cases (2)-(4), there is either a projective line or a point in $\mathbb{R} \mathbf{P}^{2}$ which is invariant under the stabilizer $G$ of $K$. In each of these cases $G$ is conjugate to one of the subgroups of $\operatorname{SL}(3, \mathbb{R})$ consisting of matrices

$$
\left[\begin{array}{lll}
* & 0 & 0 \\
* & * & * \\
* & * & *
\end{array}\right], \quad\left[\begin{array}{lll}
* & * & * \\
0 & * & * \\
0 & * & *
\end{array}\right] \text {. }
$$

In both cases, there is a homomorphism $\rho: G \rightarrow \operatorname{SL}(2, \mathbb{R})$ such that if $g \in$ $[G, G]$ then

$$
\operatorname{tr}(g)=1+\operatorname{tr}(\rho(g))
$$

(We take $g$ to lie in the commutator subgroup so as to assume that the $(1,1)$ matrix entry and the determinant of the $(2 \times 2)$-block are both 1.)

We suppose that $\phi_{n}$ is a sequence as above converging to $\phi$. Since $\phi$ is a discrete embedding, apply Lemma 2 to the restriction $h$ of $\rho \circ \phi$ to $F=[\pi, \pi]$. We deduce that there exists $\gamma \in \pi$ such that $\operatorname{tr}(\phi(\gamma))<1$. However, as discussed in $[5,3.2(3)]$, every $1 \neq \gamma \in \pi$ has the property that $\phi_{n}(\gamma) \in \operatorname{SL}(3, \mathbb{R})$ has positive eigenvalues; in particular, $\operatorname{tr} \phi_{n}(\gamma)>3$. Since $\phi_{n} \rightarrow \phi$, it follows that $\operatorname{tr} \phi(\gamma) \geq 3$, a contradiction.

Thus only case (1) is possible: $K$ is properly convex with interior $\Omega$. Then $\Gamma$ acts isometrically with respect to the Hilbert metric on $\Omega$. Since $\Gamma$ is discrete, torsionfree, and acts properly on $\Omega$, the quotient $\Omega / \Gamma$ is a closed surface. Since $\Gamma$ is not virtually abelian, $\Omega$ is not a triangular region and by [8] (see also [5, 3.2]) it follows that $\Omega / \Gamma$ is a convex $\mathbb{R} \mathbf{P}^{2}$-manifold homeomorphic to $\Sigma$. This concludes the proof of Theorem B, assuming Lemma 2.

Proof of Lemma 2. By passing to a subgroup of $F$ we may assume that the quotient of the hyperbolic plane by the image of $h(F)$ under the quotient homomorphism $\operatorname{SL}(2, \mathbb{R}) \rightarrow \operatorname{PSL}(2, \mathbb{R})$ is a complete surface which is homeomorphic to a pair-of-pants $P$ (a sphere minus three discs). Let $f_{1}, f_{2}, f_{3}$ be elements of $\pi_{1}(P) \subset F$ corresponding to the three components of $\partial P$. We choose elements $\widetilde{h\left(f_{j}\right)} \in \operatorname{SL}(2, \mathbb{R}) \quad(j=1,2,3)$ so that $\operatorname{tr}\left(\widetilde{h\left(f_{j}\right)}\right)>2$ (equivalently, $\widetilde{h\left(f_{j}\right)}$ lies in a hyperbolic one-parameter subgroup of $\left.\operatorname{SL}(2, \mathbb{R})\right)$. Now $f_{1} f_{2} f_{3}=1$ in $F$ but

$$
\widetilde{h\left(f_{1}\right)} \widetilde{h\left(f_{2}\right)} \widetilde{h\left(f_{3}\right)}=(-1)^{\chi(P)}=-1
$$

(since the relative Euler class of the representation equals -1 ; compare the discussion in $[4, \S 4])$. Since each $h\left(f_{i}\right)$ is hyperbolic, an odd number of $f_{i}$ must satisfy $\operatorname{tr}\left(h\left(f_{i}\right)\right)<2$; in particular, at least one $f \in F$ satisfies $\operatorname{tr}(h(f))<0$.

\section{REFERENCES}

1. S. Choi, Real projective surfaces, Doctoral dissertation, Princeton Univ., 1988.

2. Compact $\mathbb{R P}^{2}$-surfaces with convex boundary $\mathrm{I}: \pi$-annuli and convexity (submitted).

3. W. Goldman, Characteristic classes and representations of discrete subgroups of Lie groups, Bull. Amer. Math. Soc. (N.S.) 16 (1982), 91-94. 
4. __ Topological components of spaces of representations, Invent. Math. 93 (1988), 557607.

5. $\_$, Convex real projective structures on compact surfaces, J. Differential Geom. 31 (1990), 791-845.

6. W. Goldman and J. Millson, Local rigidity of discrete groups acting on complex hyperbolic space, Invent. Math. 88 (1987), 495-520.

7. N. J. Hitchin, Lie groups and Teichmüller space, preprint, Univ. of Warwick, 1991.

8. N. H. Kuiper, On convex locally projective spaces, Conf. Internat. Geom. Diff. Italy, 1954, pp. 200-213.

Topology and Geometry Research Center, Kyungpook National University, 702-701 TAEGU, KoREA

Department of Mathematics, University of Maryland, College Park, Maryland 20742

E-mail address:wmg@sofya.umd.edu 